\begin{tabular}{|c|l|}
\hline Title & Transient crater growth in granular targets: A n experimental study of low velocity impacts into glass sphere targets \\
\hline Author(s) & Y amamoto, Satoru; Wada, Koji; Okabe, Norihisa; Matsui, Takafumi \\
\hline Citation & $\begin{array}{l}\text { Icarus, 183(1), 215-224 } \\
\text { https://doi.org/10.1016/.icarus.2006.02.002 }\end{array}$ \\
\hline Issue Date & $2006-07$ \\
\hline Doc URL & http://hdl.handle.net/2115/14549 \\
\hline Type & article (author version) \\
\hline File Information & SY amamotoEtal.pdf \\
\hline
\end{tabular}

Instructions for use 


\title{
Transient crater growth in granular targets: An experimental study of low velocity impacts into glass sphere targets
}

\author{
${ }^{1}$ Satoru Yamamoto, ${ }^{2}$ Koji Wada, ${ }^{1}$ Norihisa Okabe, ${ }^{1}$ Takafumi Matsui \\ ${ }^{1}$ Graduate School of Frontier Sciences, University of Tokyo, Chiba 277-8562, Japan \\ ${ }^{2}$ Institute of Low Temperature Science, Hokkaido University, Sapporo 060-0819, Japan \\ Tel: +81-4-7136-3954 Fax: +81-4-7136-3954 E-mail: yamachan@impact.k.u-tokyo.ac.jp
}

Submitted to Icarus, June 2, 2005; Revised, January, 3, 2006

Number of manuscript: 17 pages

Number of figures: 10

Number of Tables: 4 
proposed running head: Transient crater growth

Send editorial communications to:

Satoru Yamamoto

Mailbox 408

Graduate School of Frontier Sciences

University of Tokyo

Kashiwanoha, Kashiwa

Chiba, 277-8562, Japan

E-mail: yamachan@impact.k.u-tokyo.ac.jp

Fax:+81-4-7136-3954 


\begin{abstract}
We experimentally studied the formation and collapse processes of transient craters. Polycarbonate projectiles with mass of $0.49 \mathrm{~g}$ were impacted into the soda-lime glass sphere target (mean diameters of glass spheres are $\sim 36,72$, and $220 \mu \mathrm{m}$, respectively) using a single-stage light-gas gun. Impact velocity ranged from 11 to $329 \mathrm{~m} \mathrm{~s}^{-1}$. We found that the transient crater collapses even at laboratory scales. The shape (diameter and depth) of the transient crater differs from that of the final crater. The depth-rim diameter ratios of the final and transient craters are 0.11-0.14 and $0.26-0.27$, respectively. The rim diameter of both the transient and final crater depends on target material properties; however, the ratio of final to transient crater diameter does not. This suggests that target material properties affect the formation process of transient craters even in the gravity regime, and must be taken into account when scaling experimental results to planetary scales. By observing impacts into glass sphere targets, we show that although the early stage of the excavation flow does not depend on the target material properties, the radial expansion of the cavity after the end of vertical expansion does. This suggests that the effect of target material properties is specifically important in the later part of the crater excavation and collapse.
\end{abstract}

Key words: (1) CRATERING (2) IMPACT PROCESSES (3) COLLISIONAL PHYSICS

\title{
1 Introduction
}

The growth of impact craters larger than a certain size, or formed in a target with negligible cohesive strength, is controlled by gravity. This is the case for impact craters on the Earth, and impacts into regolith layers on planets and satellites. Such craters are said to form in the gravity regime. The impact excavates a bowl-shaped cavity, termed the transient crater (see Fig. 1), which is subsequently modified by collapse of the transient crater rim and, in very large impact events, uplift of the crater floor. The results of rim collapse are evident at 1-4 km-diameter terrestrial impact craters (e.g., Brent, Barringer)(see e.g., Grieve et al., 1977; Melosh, 1989; Melosh and Ivanov, 1999), where lenses of rock debris, presumably from the transient crater walls, have pooled at the base of the transient crater. In this case, the rim diameter of the final crater (we call this the final rim diameter, $D_{\mathrm{fi}}$, hereafter) may be given by the sum of the rim diameter of the transient crater (we call this the transient rim diameter, $D_{\text {tr }}$, hereafter) and the change in diameter $\Delta D_{\mathrm{c}}$ due to collapse (that is, $D_{\mathrm{fi}}=D_{\mathrm{tr}}+\Delta D_{\mathrm{c}}$ ).

Many impact experiments into granular targets have been conducted to study transient cavity growth (and collapse) in the gravity regime, and how crater size scales with various impact parameters, for example velocity, impactor size, or gravity (e.g., Stöffler et al., 1975; Gault and Wedekind, 1977; Schmidt, 1980; Schmidt and Holsapple, 1982; Schmidt and Housen, 1987; Mizutani et al., 1983; Hartmann, 1985; Cintala et al., 1999; Walsh et al., 2003; Cintala et al., 2003). However, based on these previous experiments it is unclear whether or not transient crater collapse occurs at laboratory scales and, consequently, whether or not the crater radii reported in these studies are $D_{\text {tr }}$ or $D_{\mathrm{fi}}$. There are two possibilities on this issue: First, collapse may have occurred in these previous experiments, but the degrees of collapse were not large enough to be noticed (for example, in the quarter-space experiment by Schmidt and Housen (1987), the shape of the transient crater was shown to change owing to the collapse, but the change in crater diameter due to the collapse was negligible). Second, the cohesion among target particles in granular targets may be large enough to prevent collapse at laboratory scales. Therefore, the first goal of this study is to assess whether or not any collapse occurs and how extensive the collapse is.

Another goal of our study is to investigate whether or not target material properties affect the size of the transient and/or final crater. Pi-group scaling has been used as a scaling relation for impact crater sizes (e.g., Holsapple, 1993). In this analysis, two dimensionless parameters are used: $\Pi_{\mathrm{R}}\left[=(\rho / m)^{1 / 3} D_{\mathrm{fi}} / 2\right]$ (called the scaled-crater radius) and $\Pi_{2}\left(=3.22 \mathrm{ga} / \mathrm{v}_{\mathrm{i}}^{2}\right)$ (called the gravityscaled size), where $\rho$ is the bulk density of target, $m$ is the projectile's mass, $g$ is the gravitational acceleration, $a$ is the projectile's radius, and $v_{\mathrm{i}}$ is the impact velocity. Physically, $\Pi_{\mathrm{R}}$ is roughly equal to the ratio of crater to projectile radius and $\Pi_{2}$ is essentially the inverse of the Froude number. In Fig. 2 experimental data on the final crater diameter $D_{\text {fi }}$ are plotted on a $\Pi_{R}-\Pi_{2}$ diagram. As shown in Fig. 2, the experimental data do not constitute a single line but constitute several discrete linear arrays (see also Fig. 7.3 in Melosh, 1989). This suggests that $D_{\text {fi }}$ values depend on target material properties. Although internal friction and porosity in target materials 
have been proposed to affect the crater formation (e.g., Schmidt, 1980; Melosh, 1989; Housen and Holsapple, 2003), how the target material properties affect crater formation is unknown. In other words, whether $D_{\mathrm{tr}}, \Delta D_{\mathrm{c}}$ or both are affected by target material properties. In order to study the physical plausibility of these possibilities, we observe the formation and collapse processes of transient craters for various target material properties.

\section{Observation of excavation and collapse stages of transient crater}

A schematic view of our experimental setup is shown in Fig. 3. We used a polycarbonate cylinder with a hemispherical front as a projectile $(10 \mathrm{~mm}$ diameter $\times 8 \mathrm{~mm}$ length, mass of $0.49 \mathrm{~g}$, and density of $\left.1.2 \mathrm{~g} \mathrm{~cm}^{-3}\right)$. The projectile was accelerated by a single-stage light-gas gun. The impact velocities $v_{\mathrm{i}}$ ranged from 11 to $329 \mathrm{~m} \mathrm{~s}^{-1}$. The impact angle was vertical to the target surface. We used soda-lime glass spheres as targets (mean diameters $s_{\text {av }}$ are 36,72 , and $220 \mu \mathrm{m}$, respectively; these are referred as TA, TB, and TC targets hereafter). Figs. 4a-c show images of the glass spheres taken using a transmission microscope and Fig. 4d shows the number-fractions of glass spheres with diameters smaller than $s$. The bulk density, the porosity, the angle of repose, and the bulk sound speed for these targets depend on $s_{\text {av }}$ (see Table 1). The glass spheres, which filled a stainless container $(28 \mathrm{~cm}$ diameter and $11 \mathrm{~cm}$ depth), were placed in the experimental chamber. (In order to investigate the effect of the container boundary on the crater growth, we did impact experiments with a larger stainless container $(40 \mathrm{~cm}$ diameter and $15 \mathrm{~cm}$ depth). There was no significant difference between the crater diameters in the two containers for $v_{\mathrm{i}}<329 \mathrm{~m} \mathrm{~s}^{-1}$.) All the experiments were conducted at pressures lower than $50 \mathrm{~Pa}$. In order to prevent the propellant gas (Helium) from perturbing the cratering process, a deflector plate (with a hole $18 \mathrm{~mm}$ in diameter for the passage of projectiles) was set at a projectile inlet of the experimental chamber as shown in Fig. 3. The experimental condition and results are summarized in Tables 2, 3, and 4.

The formation and collapse processes of transient craters were observed by a camcorder set at a view port of the experimental chamber (Fig. 3). We took images at a time interval of $1 / 30 \mathrm{~s}$ $(=0.033 \mathrm{~s})$. Due to the difficulty of recording the exact moment $(t=0)$ when the projectile touches the target surface, we defined the first image of the excavation process as $t=0.033 \mathrm{~s}$. Figs. $5 \mathrm{a}-\mathrm{d}$ show example images of the excavation processes taken by the camcorder $\left[v_{\mathrm{i}}=290 \mathrm{~m} \mathrm{~s}^{-1}\right.$ and TC target $\left.\left(s_{\mathrm{av}}=220 \mu \mathrm{m}\right)\right]$. Fig. 5a shows the early stage of the excavation process at $t=0.067$ s. The excavation cavity (darker part) and the ejecta curtain (brighter part) are visible in this image. In addition, the projectile can be seen at the center of the crater, indicated by the letter ' $\mathrm{P}$ ' (no projectile was broken in our experiments; intact projectiles were able to retrieve after the experiments). In this experiment the transient crater was formed by $t=0.133 \mathrm{~s}$ (Fig. 5b). At this time, we see that the crater rim forms (indicated by the label 'Rim') and the ejecta curtain (brighter part) moves outward. The crater then starts collapsing (Fig. 5c); the material of the rim and the inner wall slid onto the crater floor, covering the projectile at the center of the crater. After the final crater formation (Fig. 5d), we were not able to see the projectile at the crater floor.

Fig. 5e shows the temporal change in rim diameter of transient crater with $t$ (for the same experiment as Figs. 5a-d). The transient crater forms within $0.1 \mathrm{~s}$ after impact (we can not measure the rim diameters for $t<0.1 \mathrm{~s}$ ), and $D_{\mathrm{tr}}$ is about $178 \mathrm{~mm}$ in this case. The crater then starts collapsing, and $D_{\text {tr }}$ increases from $178 \mathrm{~mm}$ to about $191 \mathrm{~mm}$. Collapse finally stops at $t=0.33 \mathrm{~s}$ after impact, and the final crater is formed (the crater shape does not change further). $D_{\text {fi }}$ is about $191 \mathrm{~mm}$ in this case. It is therefore concluded that the transient crater collapses even at the laboratory scale and $D_{\mathrm{tr}}$ increases owing to the collapse of crater wall.

In Fig. 6 the ratio $D_{\mathrm{fi}} / D_{\mathrm{tr}}$ is plotted against $D_{\mathrm{tr}}$ for TA, TB, and TC targets. As shown in this figure, $D_{\mathrm{fi}} / D_{\mathrm{tr}}$ values are nearly constant: $D_{\mathrm{fi}} / D_{\mathrm{tr}}$ does not depend on $D_{\mathrm{tr}}$. The average values of $D_{\mathrm{fi}} / D_{\mathrm{tr}}$ for TA, TB, and TC targets are estimated to be $1.07 \pm 0.02,1.05 \pm 0.01$, and $1.05 \pm 0.01$, respectively. In Fig. 6 we can see no clear difference in $D_{\mathrm{fi}} / D_{\mathrm{tr}}$ between TA, TB, and TC targets. This may suggest that $D_{\mathrm{fi}} / D_{\mathrm{tr}}$ value does not depend on target material properties. However, this does not mean that the collapse process is unaffected by target material properties; the collapse process could be controlled by internal friction. As will be shown later (Fig. 8a), $D_{\operatorname{tr}}$ values as well as $D_{\text {fi }}$ values depend on target material properties. Thus, the dependences in $D_{\text {fi }}$ and $D_{\text {tr }}$ on 
target material properties may cancel out each other. This may suggest that both excavation and collapse processes depend on target material properties in the same way, implying that the physics of the late stages of excavation is similar to that involved during collapse.

For a comparison, we also did additional experiments with dry sand (with grain diameter ranging from 200 to $500 \mu \mathrm{m}$, the angle of repose of $\sim 32^{\circ}$, and the bulk density of $1.44 \mathrm{~g} \mathrm{~cm}^{-3}$ ). However, we did not see clear evidence of collapse (e.g., sliding of the material of crater rim and inner wall) for the dry sand. This may suggest that the collapse is very dependent on the type of target, and may explain why the effects of collapse were not clear in the previous experiments.

\section{Relation between depth and diameter of crater}

Next we studied the relation between the crater depth and rim diameter (depth-rim diameter ratio) for the transient and final craters. It is difficult to measure the depths of the transient craters from the images. Therefore, we estimate the depth $h_{\mathrm{tr}}$ of the transient crater as follows: As shown in Figs. 5a and 5b, the projectile can be observed at the base of the transient crater. Thus, in this study we defined the distance between the backside of the projectile and the original target surface as $h_{\mathrm{tr}}$.

In Fig. $7 h_{\mathrm{tr}}$ is plotted against $D_{\mathrm{tr}}$ for TA, TB, and TC targets, in which shows that $h_{\mathrm{tr}}$ values for TA, TB, and TC targets are nearly proportional to $D_{\mathrm{tr}}$. Calculating the slopes of the best fit lines gives approximate depth-rim diameter ratios $r_{\mathrm{tr}}\left(=h_{\mathrm{tr}} / D_{\mathrm{tr}}\right)$ for transient craters formed in TA, TB, and TC targets. They are $0.27,0.26$, and 0.26 , respectively, which are all slightly larger than those for the previous experiments: 0.14-0.25 for the quartz sand (Gault and Wedekind, 1977; Mizutani et al., 1983) and $\sim 0.23$ for the coarse-grained sand (Cintala et al., 1999). However, this does not mean necessarily that the transient craters in this work are deeper than those in the previous experiments, because the data of the previous works are for the final craters (in the quarter-space experiments by Schmidt and Housen (1987) and Schultz (2003), the shapes of the transient craters were deeper than those of the final craters).

For comparison, $h_{\mathrm{f}}$ is also plotted against the final rim diameter $D_{\mathrm{fi}}$ for TA, TB, and TC targets in Fig. 7, where $h_{\mathrm{fi}}$ is the depth of the final crater measured from the original target surface. From this we see that $h_{\mathrm{fi}}$ values for TA, TB, and TC targets are nearly proportional to $D_{\mathrm{fi}}$ values, and the final crater depth-rim diameter ratios $\left(r_{\mathrm{fi}}=h_{\mathrm{fi}} / D_{\mathrm{fi}}\right)$ for TA, TB, and TC targets are $0.14,0.11$, and 0.11 , respectively. These $r_{\mathrm{fi}}$ values are smaller than those of the previous studies: $0.14-0.25$ for the quartz sand (Gault and Wedekind, 1977; Mizutani et al., 1983) and 0.23 for the coarsegrained sand (Cintala et al., 1999). This means that the shapes of final craters in the glass sphere targets are shallower than those in the quartz sand and coarse-grained sand. The reason for this is hard to explain, but is probably not due to differences in the angles of response, because the angles of repose for the glass spheres used in our experiments, which ranged from $25-33^{\circ}$, are not substantially different from that of coarse-grained sand used by previous workers (e.g., $32^{\circ}$; Cintala et al., 1999). The sphericity of the grains may be important; the shape of the glass sphere (Fig. 4 ) is obviously smoother than angular sand, so that the glass spheres might have lower dynamical coefficient of friction.

\section{Effect of material properties on formation of transient craters}

\subsection{Observation}

In Fig. 8a $D_{\mathrm{tr}}$ is plotted against $v_{\mathrm{i}}$. As shown clearly in this figure, $D_{\mathrm{tr}}$ increases with increasing $v_{\mathrm{i}}$. The data for TA, TB and TC targets suggest the power-law relations (broken lines in Fig. 8a): $D_{\mathrm{tr}}=(21.8 \pm 0.7) v_{\mathrm{i}}^{0.344 \pm 0.007}, D_{\mathrm{tr}}=(23.8 \pm 0.6) v_{\mathrm{i}}^{0.354 \pm 0.006}$, and $D_{\mathrm{tr}}=(25.2 \pm 1.1) v_{\mathrm{i}}^{0.356 \pm 0.009}$, respectively. The power-law exponent increases slightly from TA to TC. It is also evident from this figure that $D_{\text {tr }}$ increases from TA to TC. As shown in Table 1, the target material properties such as the porosity or the angle of repose are different between TA, TB, and TC targets. It is thus suggested that the formation of the transient crater depends on the target material properties. 
In Fig. 8b scaled-crater radius $\Pi_{\mathrm{R}}$ for $D_{\text {tr }}$ is plotted against the gravity-scaled size $\Pi_{2}$. We see that $\Pi_{\mathrm{R}}$ increases from TA to TC. Assuming the power-law relation between $\Pi_{\mathrm{R}}$ and $\Pi_{2}$, we can determine the power-law exponents $\epsilon$ (dashed-lines in Fig. 8b) for TA, TB, and TC targets. They are $\epsilon=-0.172 \pm 0.003,-0.177 \pm 0.003$, and $-0.178 \pm 0.005$, respectively. The power-law exponent $\epsilon$ for the $\Pi_{\mathrm{R}}-\Pi_{2}$ relation decreases from TA to TC. The value of $\epsilon$ is related to the coupling parameter that links the early and late stages in impact cratering (Holsapple, 1993). It is therefore proposed that this coupling parameter also depends on target material properties.

For comparison, $\Pi_{\mathrm{R}}$ values for $D_{\mathrm{fi}}$ measured by Mizutani et al. (1983) for the quartz sand target and Hartmann (1985) for the basalt and pumice powder targets are also plotted in Fig. 8b. It is clear that $\Pi_{R}$ for Mizutani et al. (1983) and Hartmann (1985) are smaller than those for our results. If we take into account the difference between $D_{\mathrm{fi}}$ and $D_{\mathrm{tr}}$ (that is, $D_{\mathrm{fi}}>D_{\mathrm{tr}}$ ), this difference may increase. Therefore, even in the gravity regime the formation of transient craters may be affected by target material properties.

One may consider that the differences in $\Pi_{\mathrm{R}}$ between this work and the previous studies are due to the low impact velocities $\left(v_{\mathrm{i}}=11\right.$ to $\left.329 \mathrm{~m} \mathrm{~s}^{-1}\right)$ in this work. However, $v_{\mathrm{i}}$ for the quartz sand and the basalt and pumice powder targets are also low: $v_{\mathrm{i}}=158 \mathrm{~m} \mathrm{~s}^{-1}$ for basalt and pumice powder targets plotted in Fig. 8b (Hartmann, 1985) and $v_{\mathrm{i}}=35$ to $830 \mathrm{~m} \mathrm{~s}^{-1}$ for Mizutani et al. (1983). Thus, it is unlikely that the differences in $\Pi_{R}$ between this work and the previous studies are due to the difference in $v_{\mathrm{i}}$.

\subsection{Interpretation}

The above results suggested that even in the gravity regime the formation of transient craters may be affected by target material properties. In this section we consider which material properties might affect the transient crater growth and explain the differences in the $\Pi_{\mathrm{R}}-\Pi_{2}$ relation among various targets. We study the relation between $\Pi_{R}$ and the following four parameters: mean grain size, porosity, internal friction, and target-projectile density ratio.

Our experimental results suggest that grain size may affect the transient crater growth. $\Pi_{R}$ values for TA, TB, and TC targets were shown to increase with increasing the mean grain size $\left(s_{\text {av }}\right)$ (Fig. $8 \mathrm{~b}$ ). However, grain size alone can not explain the differences in $\Pi_{\mathrm{R}}$ in Fig. $8 \mathrm{~b}$, because $\Pi_{\mathrm{R}}$ values for the quartz sand are smaller than those for the TC target, although $s_{\mathrm{av}}(\sim 200 \mu \mathrm{m})$ and the size range $(\sim 100$ to $250 \mu \mathrm{m})$ for the quartz sand (Mizutani et al., 1983) are similar to those for TC target (Fig. 4). Moreover, the internal friction and porosity of the target materials used in our experiment both depend upon the grain size.

The porosity of target material has been proposed to have the most significant effect on crater growth in granular targets (e.g., Melosh, 1989; Housen and Holsapple, 2003): larger craters are formed in targets with lower porosity. We can see in Fig. $8 \mathrm{~b}$ that $\Pi_{\mathrm{R}}$ values for TA, TB, and TC targets increase with decreasing the porosity (the porosities are 40, 38, and 36\%, respectively). Thus the experimental results for TA, TB, and TC targets are consistent with this trend. However, we can also observe the exception against this trend; the data for the Ottawa sand (solid line in Fig. $8 \mathrm{~b}$ ) is smaller than those for the glass sphere targets, although the porosity for the Ottawa sand ( 31-36\%; Schmidt and Housen, 1987) is not higher than those for the glass sphere targets. (They showed only the bulk density for the Ottawa sand. Assuming the grain density of 2.6-2.8 g $\mathrm{cm}^{-3}$, we estimated the porosity.) Therefore, porosity alone can not explain the difference in $\Pi_{R}$ amongst target materials.

The internal friction (which is related to the angle of repose) has been also thought to be important for the crater formation (e.g., Schmidt, 1980): larger craters are formed in targets with lower internal friction. The experimental results for TA, TB, and TC targets are consistent with this trend: we see in Fig. $8 \mathrm{~b}$ that $\Pi_{\mathrm{R}}$ values for TA, TB, and TC targets increase with decreasing the angle of repose (the angles of repose are 33,27 , and $25^{\circ}$, respectively). Since the angles of repose were not shown in the previous experiments (Mizutani et al., 1983, Hartmann, 1985, Schmidt and Housen, 1987), we cannot conclude whether or not the angle of repose can explain the differences in $\Pi_{R}$ amongst target materials. In order to investigate this, we did additional experiments with dry sand with an angle of repose of $\sim 32^{\circ}$. The results for the dry sand are also plotted in Fig. 8b (open squares). It is clearly shown in this figure that $\Pi_{R}$ values for the dry sand are smaller than those for the glass sphere targets, although the angle of repose for the dry sand $\left(32^{\circ}\right)$ is similar to that for TA target. Thus, the angle of repose alone can not explain the difference. 
The target-projectile density ratio may play important role in the $\Pi_{\mathrm{R}}-\Pi_{2}$ relation (e.g., Holsapple, 1993; Housen and Holsapple, 2003): $\Pi_{\mathrm{R}}$ depends on $(\rho / \delta)^{\gamma}$ as well as $\Pi_{2}$, where $\rho / \delta$ is the ratio of target to projectile density and $\gamma=(2+\mu-6 \nu) /(6+3 \mu)$, where $\mu$ and $\nu$ are constants relating to the coupling parameters. The density ratio for this work $(\rho / \delta \sim 1.3)$ is larger than those for the previous experiments: $\rho / \delta \sim 0.65$ (Schmidt and Housen, 1987), 0.49 (Mizutani et al., 1983), and 0.31 to 0.74 (Hartmann, 1985). However, $\nu$ is typically $1 / 3$ and $\mu$ is about $\sim 0.4$ (Holsapple, 1993), that is, the exponent is $\gamma \sim 0.056$. In this case, the differences in $(\rho / \delta)^{\gamma}$ between this work and the previous experiments are $\sim 4 \%$ (Schmidt and Housen, 1987), 6\% (Mizutani et al., 1983), and $3-8 \%$ (Hartmann, 1985). These values are too small to explain the divergence amongst various target materials in Fig. 8b.

The above facts suggest that the differences in $\Pi_{R}$ amongst target materials can not be explained by the effect of a single parameter. The combination of these parameters may be able to explain the differences. More detailed study of the effect of target material properties on the transient crater formation is required.

\section{Observation of the excavation process}

In order to directly observe the excavation process, we conducted further impact experiments by using the quarter-space technique (e.g., Schmidt and Piekutowski, 1983; Schmidt and Housen, 1987; Schultz, 2003) in which the glass sphere target was set in a sample box with a transparent window, and projectiles impacted the target vertically along the transparent window. Figs. 9a-c show images of the excavation process taken at $t=0.033,0.067$, and $0.100 \mathrm{~s}$, respectively (the first image of the excavation process was recorded at $t=0.033 \mathrm{~s}$ ), for impact into TB target with $v_{\mathrm{i}}=$ $154 \mathrm{~m} \mathrm{~s}^{-1}$. The $x$-axis in the figure corresponds to the original target surface (bright region below the $x$-axis represents the crater cavity, and the projectile impacts the target along the $y$-axis). Based on these images, we can trace the change in shape of the cavity below the $x$-axis (Fig. 9d). It is clear from Fig. 9d that the vertical expansion of the cavity halts by $t=0.067 \mathrm{~s}$ but its radial expansion along the $x$-axis continues after vertical expansion stops. We conclude that the transient crater is formed by $t=0.100 \mathrm{~s}$, because the rim forms (represented by the arrows in Fig. 9c) by this time step, and the crater shape does not change again until the beginning of collapse of the wall.

We also studied the temporal change in the diameter of the cavity (cavity diameter $d_{\mathrm{c}}$ ) for different targets. Fig. 10 shows the temporal change in $d_{\mathrm{c}}$ for TA, TB, and TC targets $\left(v_{\mathrm{i}}=\right.$ $\left.136 \pm 3 \mathrm{~m} \mathrm{~s}^{-1}\right)$. We can see that $d_{\mathrm{c}}$ value at $t=0.033 \mathrm{~s}$ ranges from 6 to $7 \mathrm{~cm}$ and increases to about $9 \mathrm{~cm}$ at $t=0.067 \mathrm{~s}$. This feature does not differ greatly amongst TA, TB, and TC targets. However, a large difference in $d_{\mathrm{c}}$ amongst the targets appears after $t=0.067 \mathrm{~s}$ : $d_{\mathrm{c}}$ values for TB and TC targets rapidly increase after this time step, while $d_{\mathrm{c}}$ value for TA target does not. We again consider that the transient crater is formed by $t=0.100 \mathrm{~s}$, because the rim was formed by $t=0.100 \mathrm{~s}$ (Fig. 9c). Then, $d_{\mathrm{c}}$ increases slightly after $0.133 \mathrm{~s}$ (the arrow in Fig. 10). We consider that the changes in $d_{\mathrm{c}}$ after $t=0.133 \mathrm{~s}$ are due to collapse; $d_{\mathrm{c}}$ values for TA and TB targets increase about $10 \%$ due to the collapse, while the change in $d_{\mathrm{c}}$ for TC target is much smaller than this. As shown in Fig. 6, the change in rim diameter for TC target due to collapse was similar to those for TA and TB targets. However, the change for TA target in this quarter-space experiment is smaller than those for TB and TC targets. The reason for the difference is uncertain.

These observations suggest that the radial expansion process depends on the target material properties. This might be interpreted as follows: During the early stages of excavation $(t<\sim 0.067$ $\mathrm{s})$, the dynamic pressure of the excavation flow is high enough to dominate over the effects of target material properties. Therefore, the growth of the cavity does not depend on target material properties. On the other hand, the dynamic pressure of the flow decreases at the stage after 0.067 $\mathrm{s}$ where the radial expansion process dominates. Thus, the excavation flow would be perturbed by material properties such as internal friction. This could be the reason why the transient rim diameters depend on the target material properties even in the gravity regime. 


\section{Conclusions}

We observed the formation and collapse of transient craters formed in glass-sphere targets with different target material properties. We found that the transient crater collapses even at laboratory scales, and that the shape of the final crater was broader and shallower than the transient crater. The depth-rim diameter ratios of the final and transient craters were $0.11-0.14$ and $0.26-0.27$, respectively. The increases in crater diameter due to the collapse were about 3 to $12 \%$, but did not show any dependency on the target material properties. On the other hand, both the transient and final rim diameters depend on the target material properties. This means that the formation of transient craters is affected by target material properties in the gravity regime. Clearly there is a need to take into account the effect of target material properties on the scaling laws for transient craters in the gravity regime. Observations of the excavation flow in impact experiments using the quarter-space technique, suggest that the early stage of crater growth does not depend on the target material properties, whereas the later radial expansion does.

The feature of the collapse processes observed in this study may be analogous to the formation of breccia lenses observed at the base of craters (such as Brent and Barringer) (e.g., Grieve et al., 1977; Melosh, 1989; Melosh and Ivanov, 1999); the breccia lenses are mainly due to debris sliding of the rim and wall of transient craters. The slumping of the crater rim in the case of simple craters was also shown in numerical calculations (e.g., Ivanov, 1999; Wünnemann and Ivanov, 2003; Wada et al., 2006). Therefore, combining our data (such as the diameter ratio of final to transient crater or the depth-rim diameter ratio) with numerical calculations and field studies will provide more information about the understanding of the formation of simple craters with breccia lenses.

\section{Acknowledgments}

The authors thank O.S. Barnouin-Jha, H. Senshu, S. Sugita, and T. Kadono for fruitful discussions, G. Collins and an anonymous reviewer for helpful comments, K. Barnouin-Jha for improving the draft, and S. Imanishi for technical support. This research was partly supported by T. Yoda and the Grant in Aid from Japan Society for the Promotion of Science.

\section{References}

[1] Cintala, M.J., Berthoud L., Hörz F., 1999, Ejection-velocity distributions from impacts into coarse-grained sand. Meteorit. Planet. Sci. 34, 605-623.

[2] Cintala, M.J., Barnouin-Jha O.S., Hörz F., 2003, A method of estimating transient-cavity diameters for impact craters formed in dry sand. Lunar Planet. Sci. Conf. 36th, abstract 207.

[3] Gault, D.E., Wedekind J.A., 1977, Experimental hypervelocity impact into quartz sand-II, Effects of gravitational acceleration. In: Roddy, D.J., Pepin, R.O., Merrill, R.B. (Eds.), Impact and explosion cratering, Pergamon press, New York, pp. 1231-1244.

[4] Grieve, R.A.F., Dence M.R., Robertson P.B., 1977, Cratering processes: As interpreted from the occurrence of impact melts. In: Roddy, D.J., Pepin, R.O., Merrill, R.B. (Eds.), Impact and explosion cratering, Pergamon press, New York, pp. 791-814.

[5] Hartmann, W.K., 1985, Impact experiments. 1. Ejecta velocity distributions and related results from regolith targets. Icarus 63, 69-98.

[6] Holsapple, K.A., 1993, The scaling of impact processes in planetary sciences. Annu. Rev. Earth Planet. Sci. 21, 333-373.

[7] Housen, K.R., Holsapple K.A., 2003. Impact cratering on porous asteroids. Icarus 163, 102-119.

[8] Ivanov, B.A., 1999, Transient crater modification due to gravity slumping. Lunar Planet. Sci. Conf. 30th, abstract 157.

[9] Melosh, H.J., 1989, Impact Cratering. Oxford Univ. Press, New York.

[10] Melosh, H.J., Ivanov B.A., 1999, Impact crater collapse. Annu. Rev. Earth Planet. Sci. 27, 385-415. 
[11] Mizutani, H., Kawakami S., Takagi Y., Kato M., Kumazawa M., 1983, Cratering experiments in sands and a trial for general scaling law. J. Geophys. Res. 88, Supplement, A835-A845.

[12] Schmidt, R.M., 1980, Meteor Crater: Energy of formation-implications of centrifuge scaling. Proc. Lunar Planet. Sci. Conf. 11th, 2099-2128.

[13] Schmidt, R.M., Holsapple K.A., 1982, Estimates of crater size for large-body impact: Gravityscaling results. Geol. Soc. of Am. Special Paper 190, 93-102.

[14] Schmidt, R.M., Piekutowski A.J., 1983, Development of the quarter-space technique for cratering experiments of a centrifuge. Lunar Planet. Sci. Conf. 14th, abstract 668-669.

[15] Schmidt, R.M., Housen K.R., 1987, Some recent advances in the scaling of impact and explosion cratering. Int. J. Impact Engng 5, 543-560.

[16] Schultz, P.H., 2003, Transient crater growth in low density targets. Lunar Planet. Sci. Conf. 36 th, abstract 206 .

[17] Stöffler, D., Gault D.E., Wedekind J., Polkowski G., 1975, Experimental hypervelocity impact into quartz sand: Distribution and shock metamorphism of ejecta. J. Geophys. Res. 80, 40624077.

[18] Teramoto, K., Yano H., 2005, Measurements of sound speed in granular materials simulated regolith. Lunar Planet. Sci. Conf. 36th, abstract 1856.

[19] Wada, K., Senshu H., Matsui T, 2006, Numerical simulation of impact cratering on granular material. Icarus, 180, 528-545.

[20] Walsh, A.M., Holloway K.E., Habdas P., de Bruyn J.R., 2003, Morphology and scaling of impact craters in granular media. Phys. Rev. Lett. 91, 104301.

[21] Wünnemann, K., Ivanov B.A., 2003, Numerical modelling of the impact crater depth-diameter dependence in an acoustically fluidized target. Planet. Space Sci. 51, 831-845. 


\section{Figure Captions}

Fig. 1: Schematic diagram of the collapse of a transient crater. (a) The wall and rim of the transient crater collapse inward owing to gravity, and (b) the collapsed material fills the crater floor (the dashed-line represents the original transient crater shape). In this figure we do not consider the uplift of a crater floor owing to rebound.

Fig. 2: The scaled-crater radius $\Pi_{\mathrm{R}}\left[=(\rho / m)^{1 / 3} D_{\mathrm{fi}} / 2\right]$ is plotted against the gravity-scaled size $\Pi_{2}\left(=3.22 g a / v_{\mathrm{i}}^{2}\right)$ for granular target experiments, where $D_{\mathrm{fi}}$ is the rim diameter of final crater, $\rho$ is the bulk density of target, $m$ is the projectile's mass, $g$ is the gravitational acceleration, $a$ is the projectile's radius, and $v_{\mathrm{i}}$ is the impact velocity. The broken line represents a power-law relation between $\Pi_{\mathrm{R}}$ and $\Pi_{2}$ for the data measured by Schmidt and Housen (1987). Schmidt (1980) and Schmidt and Housen (1987) measured the apparent diameters of final craters $\left(d_{\mathrm{fi}}\right)$, but they did not show the data on $D_{\mathrm{fi}}$. In this plot, we assumed $D_{\mathrm{fi}}=1.25 d_{\mathrm{fi}}$ (Gault and Wedekind, 1977) (the original power-law relation between $\Pi_{\mathrm{R}}$ and $\Pi_{2}$ for $d_{\mathrm{fi}}$ is also plotted as the dotted-line). The data on $D_{\text {fi }}$ for soda-lime glass spheres are the results of this work for $s_{\mathrm{av}}=220,72$, and $36 \mu \mathrm{m}$, where $s_{\mathrm{av}}$ is the mean diameter of glass spheres.

Fig. 3: Schematic diagram (side view) of our experimental setup. We can observe the formation and collapse processes of transient craters by a camcorder set at a view port of the experimental chamber.

Fig. 4: Example images of the glass spheres for (a) TA, (b) TB, and (c) TC targets (see text for description of targets). The scale bars are $500 \mu \mathrm{m}$ long. (d) Number-fractions of the glass spheres with diameter smaller than $s$ for TA, TB, and TC targets, respectively.

Fig. 5: Example images of the formation and collapse processes of the transient crater at (a) $t=0.067$ s, (b) $0.133 \mathrm{~s}$, (c) $0.200 \mathrm{~s}$, and (d) $1.000 \mathrm{~s}$ after impact. We can see the projectile at the center of the crater (indicated by the letter ' $\mathrm{P}$ '). Light sources illuminate the target surface from the top and the right of this picture. This is the case for impact into TC target (impact velocity of $290 \mathrm{~m} \mathrm{~s}^{-1}$ ). (e) The crater rim diameter is plotted against the time $t$ after impact.

Fig. 6: The ratio of final rim diameter $\left(D_{\mathrm{fi}}\right)$ to transient rim diameter $\left(D_{\mathrm{tr}}\right)$ is plotted against $D_{\mathrm{tr}}$.

Fig. 7: The relation between the crater depth $\left(h_{\mathrm{tr}}, h_{\mathrm{fi}}\right)$ and the rim diameter $\left(D_{\mathrm{tr}}, D_{\mathrm{fi}}\right)$ for the transient and final craters for TA, TB, and TC targets. The broken lines are the best fit ones for TA, TB, and TC targets (the number indicates the depth-rim diameter ratio: $h_{\mathrm{tr}} / D_{\mathrm{tr}}$ for the transient craters and $h_{\mathrm{fi}} / D_{\mathrm{fi}}$ for the final craters).

Fig. 8: (a) The transient rim diameter $D_{\mathrm{tr}}$ is plotted against the impact velocity $v_{\mathrm{i}}$. The broken lines represent the least-squares fit to the data for TA, TB and TC targets, respectively. (b) $\Pi_{\mathrm{R}}-\Pi_{2}$ plot for $D_{\mathrm{tr}}$ observed for TA, TB, and TC targets. The dashed lines represent the least-squares fit to the data for TA, TB, and TC targets, respectively. In order to see the difference between $D_{\mathrm{tr}}$ and $D_{\mathrm{fi}}$ in this diagram, $\Pi_{\mathrm{R}}$ values for $D_{\mathrm{fi}}$ in this work are also plotted (upper error bar for each data). For comparison, $\Pi_{\mathrm{R}}$ values for $D_{\mathrm{fi}}$ by Mizutani et al. (1983) (quartz sand) and Hartmann (1985) (basalt and pumice powders) are also plotted. The solid line represents a power-law relation between $\Pi_{\mathrm{R}}$ and $\Pi_{2}$ for the data on $D_{\mathrm{fi}}$ by Schmidt and Housen (1987) (they measured the apparent diameters of final craters $\left(d_{\mathrm{fi}}\right)$, but did not show the data on $D_{\text {fi }}$. In this plot, we assume $D_{\text {fi }}=1.25 d_{\mathrm{fi}}$ ). For a comparison, the data on $D_{\mathrm{fi}}$ for dry sand (with grain diameter ranging from 200 to $500 \mu \mathrm{m}$, the angle of repose of $\sim 32^{\circ}$, and the bulk density of $1.44 \mathrm{~g} \mathrm{~cm}^{-3}$ ) measured in this work are also plotted (open squares).

Fig. 9: Images of growth stages of cavities due to excavation flows at (a) $t=0.033 \mathrm{~s}$, (b) $0.067 \mathrm{~s}$, and (c) $0.100 \mathrm{~s}$ after impact. The $x$-axis corresponds to the original target surface. The projectile impacts the target (TB) along the $y$-axis (impact velocity of $154 \mathrm{~m} \mathrm{~s}^{-1}$ ). (d) Contours of the cavities below the $x$-axis for three images (a)-(c).

Fig. 10: Temporal change in the diameter (along the $x$-axis in Fig. 9) of the cavity for TA, TB, and TC targets. The impact velocities are $136 \pm 3 \mathrm{~m} \mathrm{~s}^{-1}$. 
Table 1: Material properties of the glass sphere targets

\begin{tabular}{lccccc}
\hline Target & $\begin{array}{c}\text { Mean diameter } \\
s_{\text {av }}[\mu \mathrm{m}]\end{array}$ & $\begin{array}{c}\text { Bulk density } \\
{\left[\mathrm{g} \mathrm{cm}^{-3}\right]}\end{array}$ & $\begin{array}{c}\text { Porosity } \\
{[\%]}\end{array}$ & $\begin{array}{c}{ }^{a} \text { Angle of } \\
\text { repose }\left[{ }^{\circ}\right]\end{array}$ & $\begin{array}{c}\text { Sound speed } \\
{\left[\mathrm{m} \mathrm{s}^{-1}\right]}\end{array}$ \\
\hline TA & 36 & 1.50 & 40 & 33 & $93 \pm 13$ \\
TB & 72 & 1.54 & 38 & 27 & $134 \pm 11$ \\
TC & 220 & 1.59 & 36 & 25 & $171 \pm 18$ \\
\hline
\end{tabular}

${ }^{a}$ The base angle of the cone-shape of the glass spheres above a flat and level plate. ${ }^{b}$ These values are from Teramoto and Yano (2005).

\section{Table}

Table 2: The experimental condition and the result for TA target.

\begin{tabular}{cccccc}
\hline Shot No. & $\begin{array}{c}\text { Impact velocity } \\
{\left[\mathrm{m} \mathrm{s}^{-1}\right]}\end{array}$ & \multicolumn{2}{c}{ Transient crater } & \multicolumn{2}{c}{ Final crater } \\
& rim diameter $[\mathrm{mm}]$ & depth $[\mathrm{mm}]$ & rim diameter $[\mathrm{mm}]$ & depth $[\mathrm{mm}]$ \\
\hline 411081 & 129 & 113 & 31 & 127 & 14 \\
411082 & 86 & 103 & 26 & 111 & 13 \\
411083 & 52 & 86 & 20 & 90 & 12 \\
411084 & 69 & 94 & 24 & 100 & 13 \\
411085 & 52 & 85 & 23 & 89 & 18 \\
411091 & 147 & 121 & 33 & 128 & 10 \\
411092 & 28 & 69 & 18 & 72 & 20 \\
411093 & 174 & 130 & 34 & 138 & 22 \\
411094 & 219 & 143 & 37 & 149 & 25 \\
411096 & 250 & 144 & 39 & 156 & 26 \\
411097 & 298 & 150 & 43 & 158 & 7 \\
411098 & 321 & 158 & 46 & 164 & 14 \\
411099 & 13 & 51 & 13 & 55 & 113 \\
4110910 & 85 & 105 & 26 & 118 & \\
4110911 & 109 & 108 & 28 & & \\
\hline
\end{tabular}


Table 3: The experimental condition and the result for TB target.

\begin{tabular}{cccccc}
\hline Shot No. & $\begin{array}{c}\text { Impact velocity } \\
{\left[\mathrm{m} \mathrm{s}^{-1}\right]}\end{array}$ & \multicolumn{2}{c}{ Transient crater } & \multicolumn{2}{c}{ Final crater } \\
& rim diameter $[\mathrm{mm}]$ & depth $[\mathrm{mm}]$ & rim diameter $[\mathrm{mm}]$ & depth $[\mathrm{mm}]$ \\
\hline 411051 & 219 & 159 & 44 & 170 & 18 \\
411052 & 96 & 122 & 29 & 128 & 15 \\
411053 & 52 & 96 & 23 & 100 & 12 \\
411054 & 51 & 98 & 24 & 101 & 12 \\
411055 & 80 & 110 & 30 & 119 & 14 \\
411056 & 39 & 86 & 22 & 92 & 12 \\
411061 & 63 & 101 & 22 & 106 & 17 \\
411062 & 179 & 149 & 40 & 158 & 16 \\
411063 & 140 & 142 & 37 & 147 & 23 \\
411064 & 329 & 181 & 49 & 188 & 21 \\
411065 & 298 & 174 & 46 & 184 & 16 \\
411066 & 266 & 174 & 44 & 180 & 14 \\
411067 & 118 & 128 & 31 & 135 & 7 \\
411068 & 110 & 128 & 31 & 136 & \\
411069 & 11 & 55 & 12 & 58 & \\
\hline
\end{tabular}

Table 4: The experimental condition and the result for TC target.

\begin{tabular}{cccccc}
\hline Shot No. & $\begin{array}{c}\text { Impact velocity } \\
{\left[\mathrm{m} \mathrm{s}^{-1}\right]}\end{array}$ & \multicolumn{2}{c}{ Transient crater } & \multicolumn{2}{c}{ Final crater } \\
& rim diameter $[\mathrm{mm}]$ & depth $[\mathrm{mm}]$ & rim diameter $[\mathrm{mm}]$ & depth $[\mathrm{mm}]$ \\
\hline 411021 & 260 & 183 & 49 & 195 & 19 \\
411022 & 139 & 148 & 41 & 155 & 16 \\
411023 & 20 & 71 & 14 & 75 & 160 \\
411024 & 152 & 153 & 42 & 204 & 21 \\
411025 & 313 & 191 & 49 & 130 & 13 \\
411031 & 88 & 123 & 28 & 139 & 14 \\
411032 & 99 & 134 & 32 & 140 & 13 \\
411033 & 101 & 132 & 36 & 169 & 23 \\
411034 & 174 & 161 & 39 & 169 & 13 \\
411035 & 202 & 162 & 47 & 121 & 10 \\
411036 & 66 & 116 & 26 & 99 & 11 \\
411041 & 44 & 96 & 24 & 95 & 19 \\
411044 & 38 & 90 & 19 & 145 & 24 \\
411046 & 114 & 139 & 38 & 189 & 191 \\
411047 & 231 & 179 & 45 & & \\
510295 & 290 & 178 & 42 & & \\
\hline
\end{tabular}


Figure 1 (Yamamoto et al.)
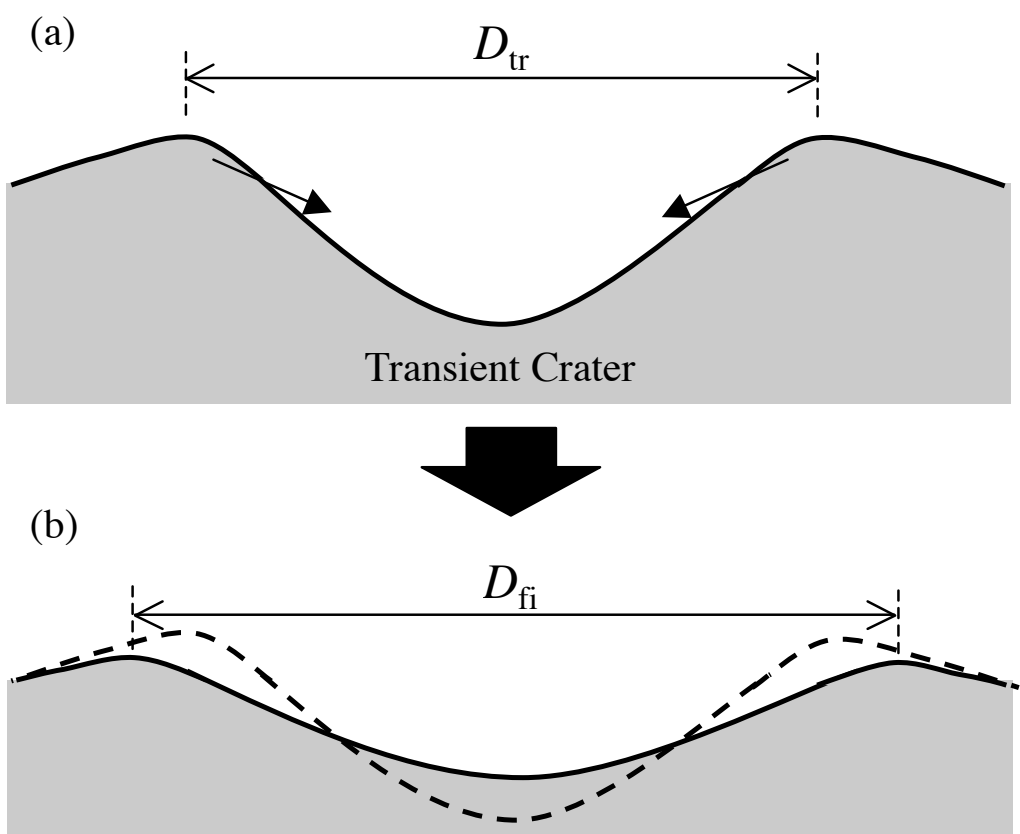

Final Crater 
Figure 2 (Yamamoto et al.)

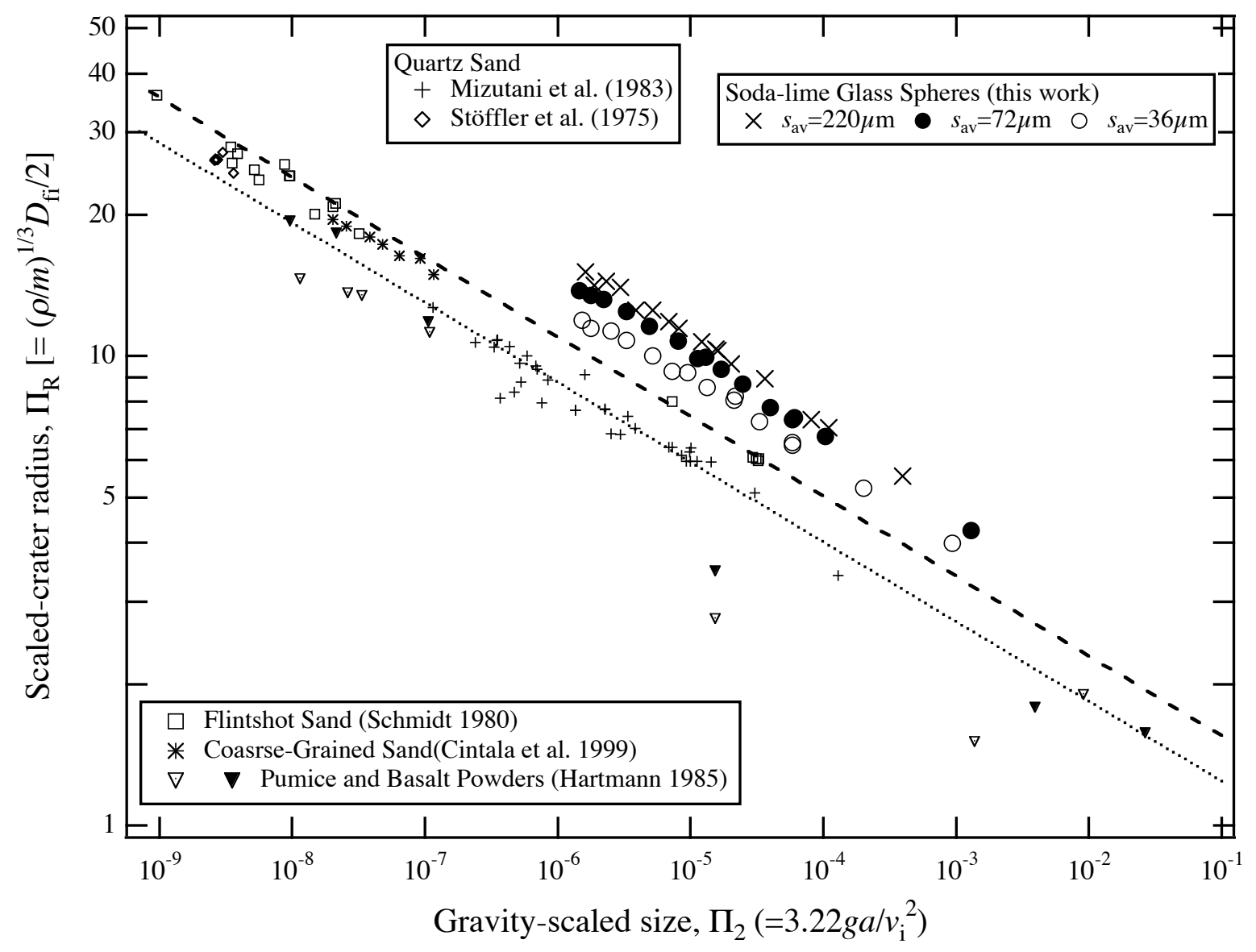


Figure 3 (Yamamoto et al. )

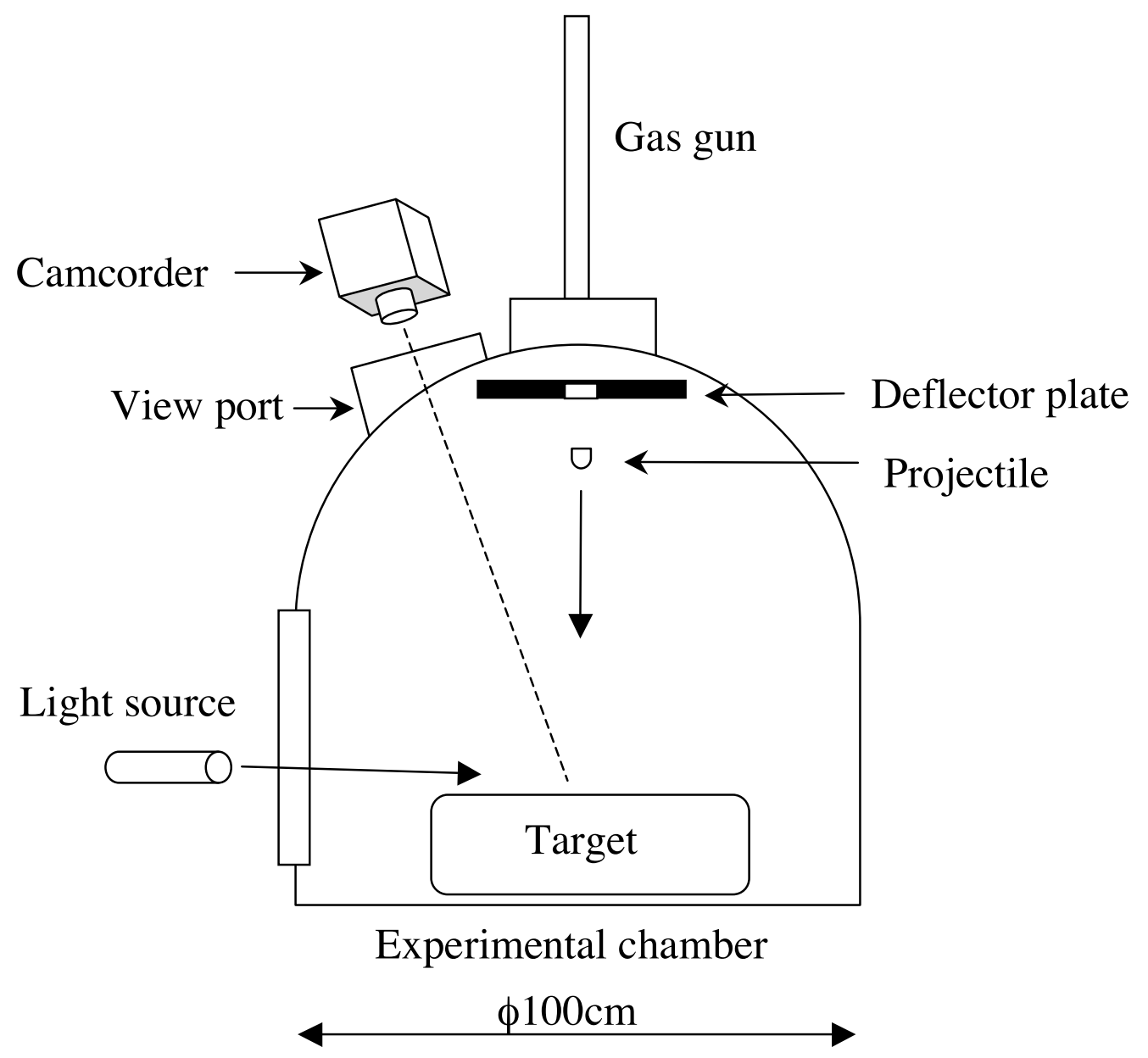


Figure 4 (Yamamoto et al.)
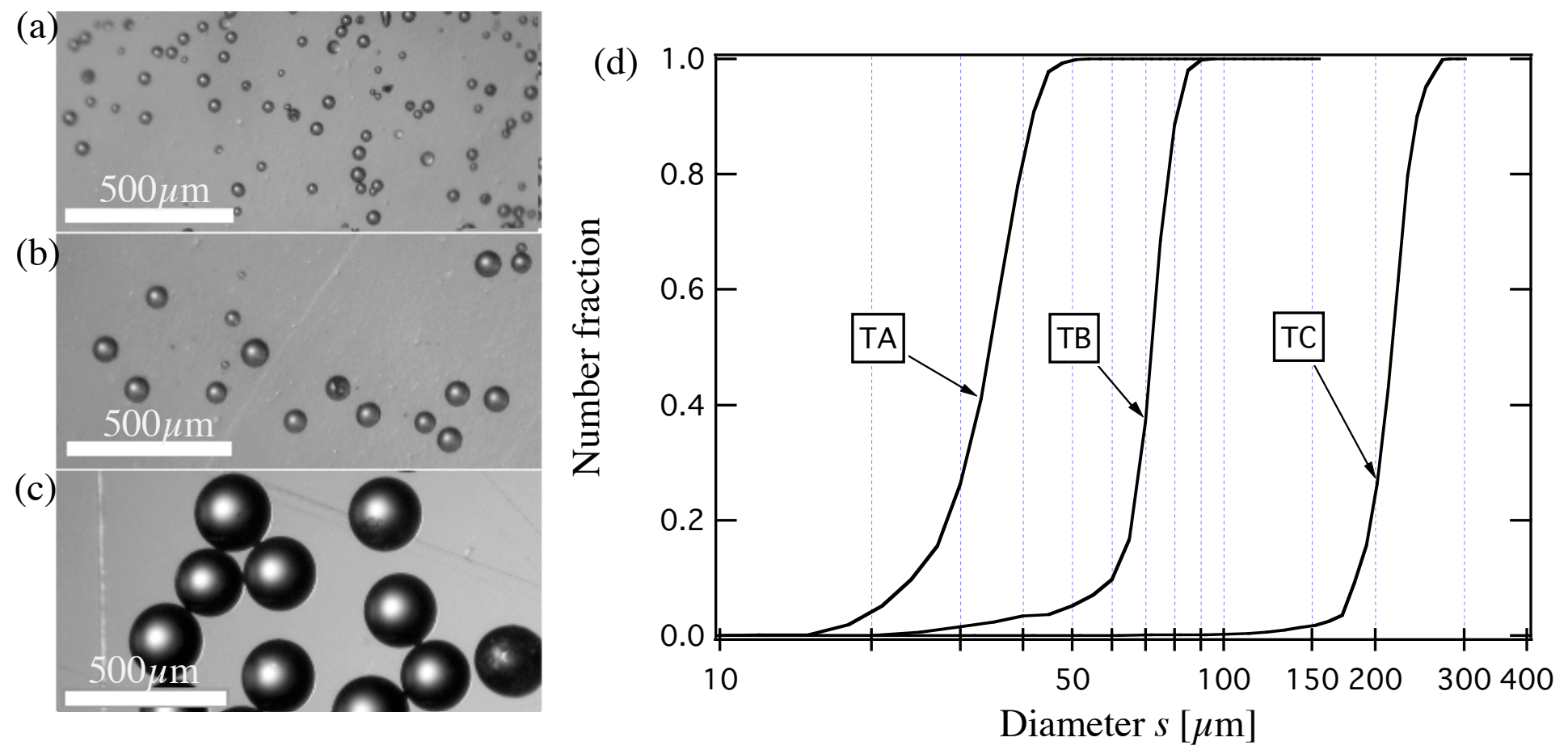
Figure 5 (Yamamoto et al.)
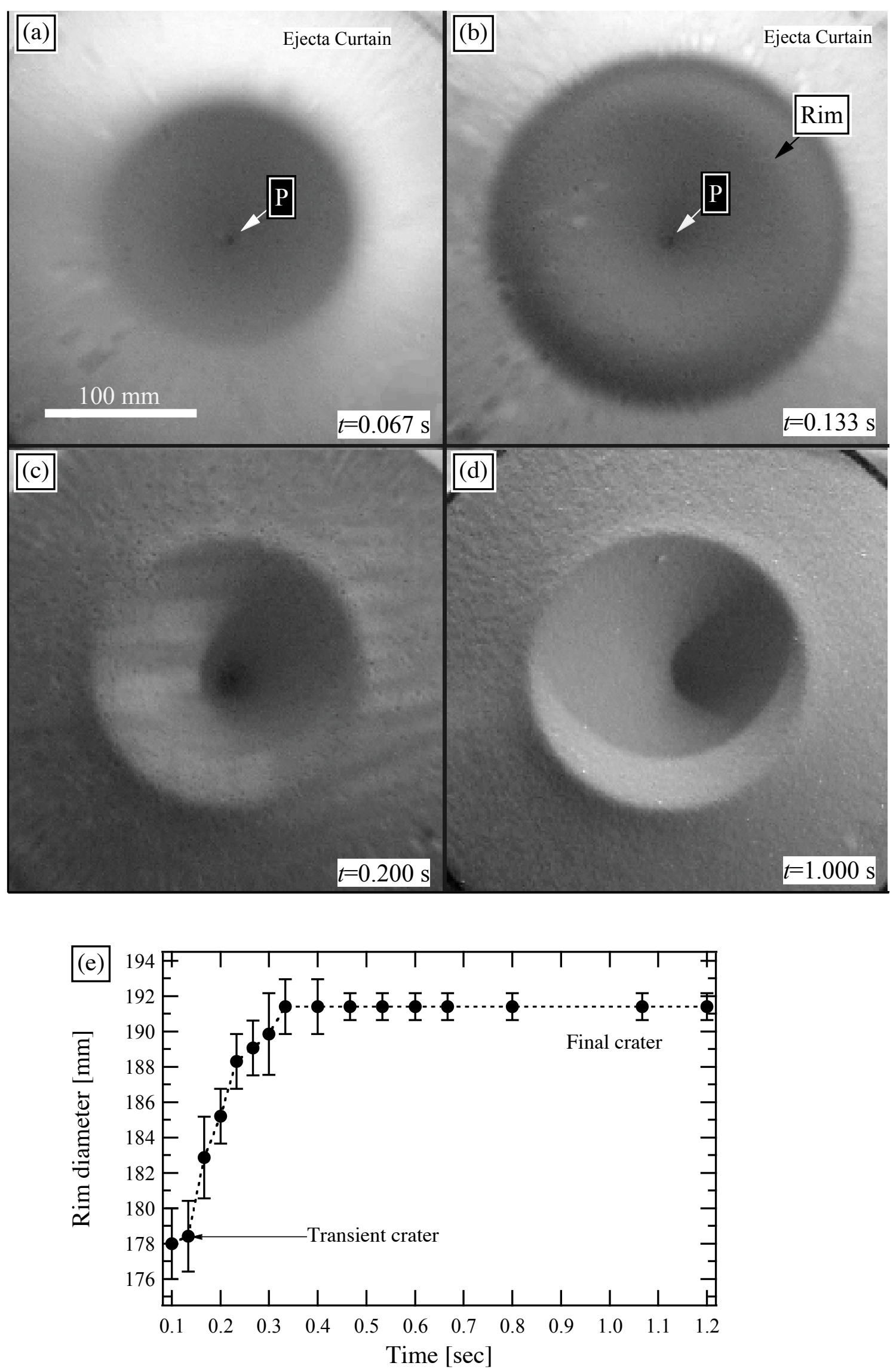
Figure 6 (Yamamoto et al.)

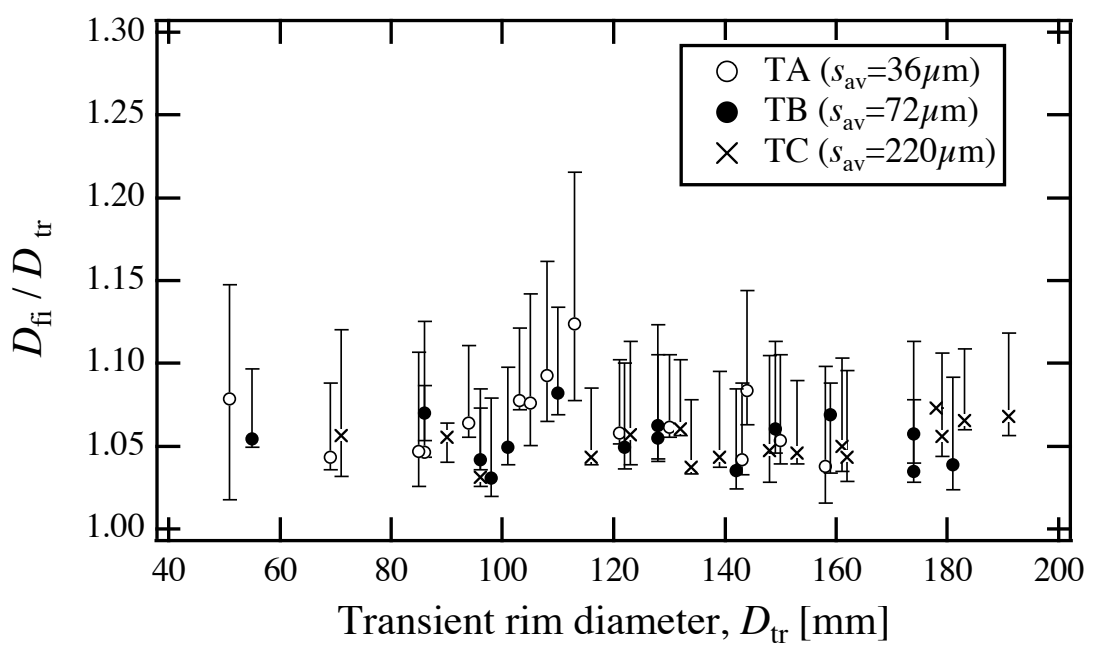


Figure 7 (Yamamoto et al.)

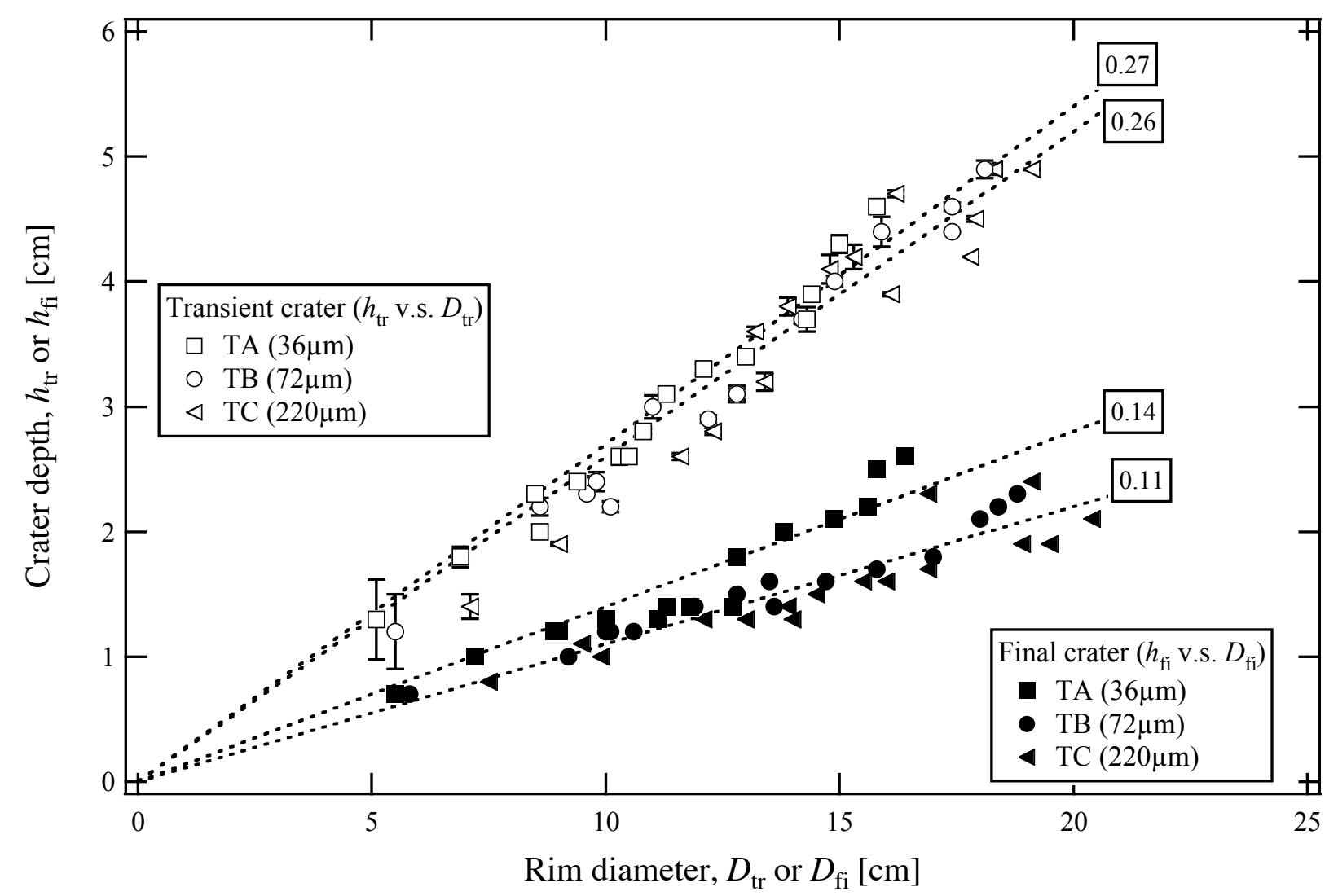


Figure 8 (Yamamoto et al.)
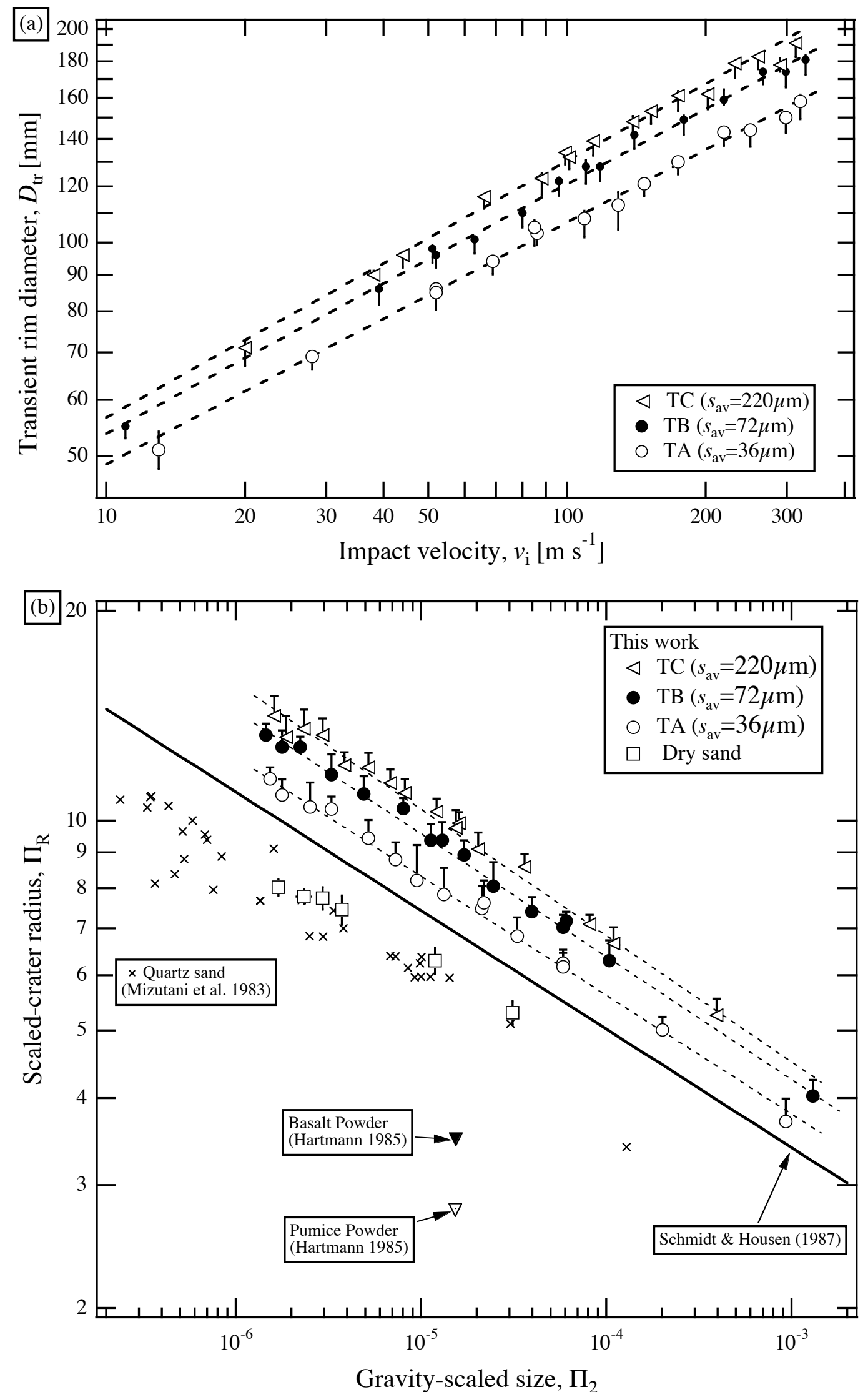
Figure 9 (Yamamoto et al.)

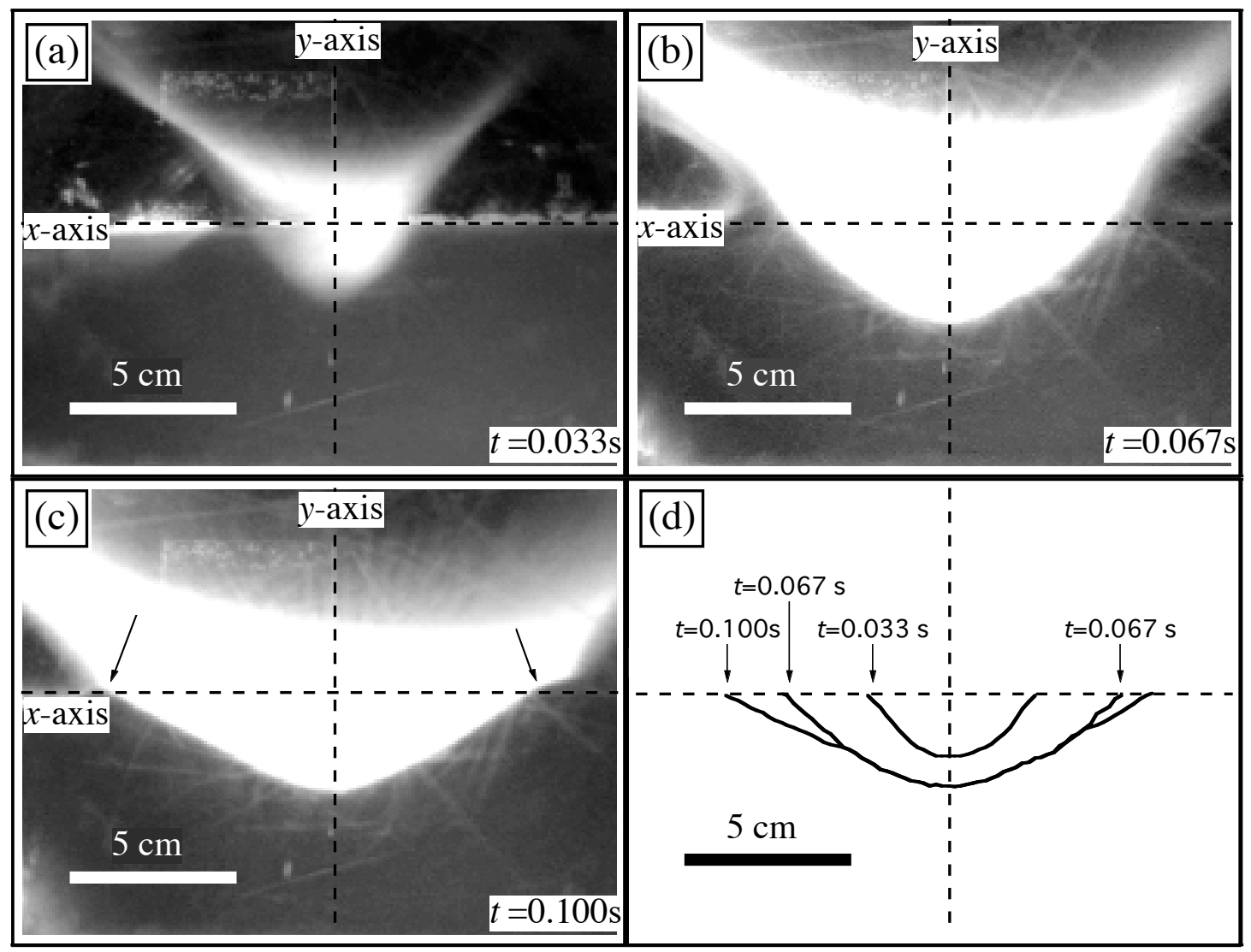


Figure 10 (Yamamoto et al.)

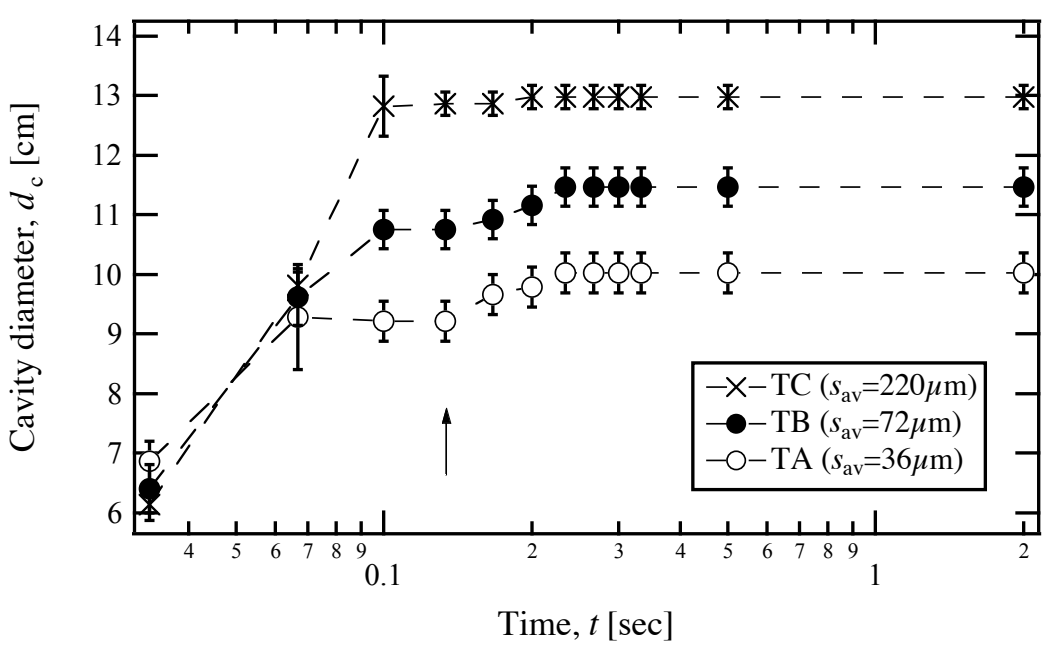

